\title{
DEVELOPMENT OF A WAKE VORTEX SPACING SYSTEM FOR AIRPORT CAPACITY ENHANCEMENT AND DELAY REDUCTION
}

\author{
David A. Hinton, Cornelius J. O'Connor \\ NASA Langley Research Center, Hampton, VA 23681
}

\section{AVOSS Overview}

A number of factors lead to a reduction in airport capacity in weather conditions that prevent the use of visual approach procedures. These factors include a reduction in the number of available runways and the longitudinal wake turbulence separation constraints used by Air Traffic Control (ATC). These wake constraints (table 1) evolved over time to prevent wake encounters in weather conditions most conducive to long-lived wakes, and are unnecessarily large in weather domains that lead to rapid wake decay or drift away from the flight path. During visual conditions aircraft separation responsibility belongs to the pilots, who use their knowledge of weather conditions, lead aircraft type, and lead aircraft flight path to effectively self-separate from wake encounters. In many situations the resulting spacing is less than would be required in instrument operations [1]. The AVOSS is designed to structure this process and minimize the difference in aircraft spacing between visual and instrument operations. The operational concept of AVOSS is to determine the spacing required to prevent wake vortex encounters, given the ambient weather conditions in existence at the airport.
The basic AVOSS architecture is unchanged from previous descriptions $[2,3,4,5$, $6]$ and shown in figure 1 . The AVOSS system uses sensed weather information to predict wake vortex behavior and develop safe spacing criteria. The meteorological subsystem uses sensors and modeling techniques to describe the vertical profiles of the wind, turbulence, and temperature from the surface to the glide slope intercept altitude. A statistical description of relevant variables is provided to minimize spatial effects and permit prediction of the worst-case wake behavior that may occur during an operational time period. The wake predictor

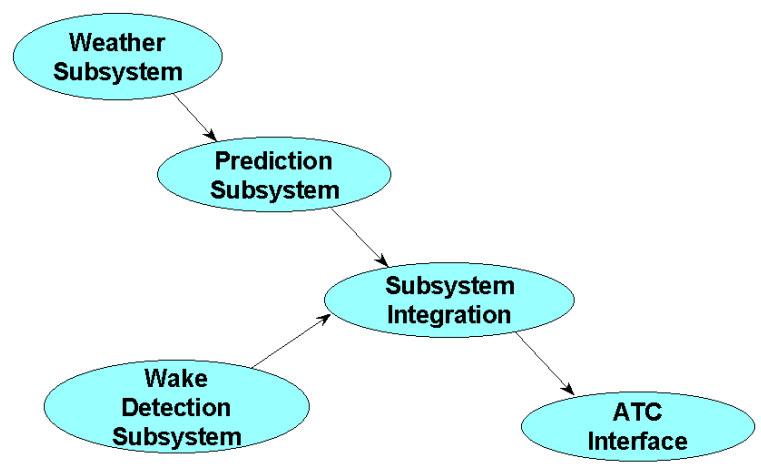

Figure 1 - AVOSS Architecture

Table 1 - FAA spacing criteria at runway threshold (NM)

\begin{tabular}{|c|c|c|c|c|}
\hline \multirow{2}{*}{ Following Aircraft } & \multicolumn{4}{|c|}{ Leading (Generating) Aircraft } \\
\cline { 2 - 5 } & Small & Large & B757 & Heavy \\
\hline Small $(<41,000 \mathrm{lb})$ & 2.5 & 4 & 5 & 6 \\
\hline $\begin{array}{c}\text { Large }(41,000 \text { to } \\
255,000 \mathrm{lb})\end{array}$ & 2.5 & 2.5 & 4 & 5 \\
\hline Heavy $(>255,000 \mathrm{lb})$ & 2.5 & 2.5 & 4 & 4 \\
\hline
\end{tabular}


uses this weather profile and descriptions of the aircraft fleet at the airport to predict wake drift rate, sink rate, and decay rate for each modeled aircraft type. The wake behavior is compared to the dimensions of a pre-defined safety corridor that extends from the glide slope intercept point to the runway. Wake behavior at a series of locations, referred to as windows, along the approach path are modeled. The time that the wake remains in the corridor (residence time) is determined at each corridor window and used to derive required aircraft separation intervals. The wake can exit the corridor by one of three wake behavior factors; either by sinking below the corridor floor, drifting outside the lateral limits, or decaying. The results at each window are combined to provide the spacing required at the top of the approach to meet both the wake vortex safety requirements and minimum threshold spacing for runway occupancy time considerations.

Wake sensors quantify the wake residence time at the approach window being scanned to verify that the wakes are behaving within the range of predicted values. The difference between the predicted and observed residence time is referred to as a prediction buffer, and negative buffers are referred to as exceedances. Within the current concept demonstration system, the exceedances are primarily a diagnostic tool and do not necessarily indicate an unsafe prediction. For example, an exceedance may be declared even when the wake vortex is behaving as predicted, if the sensor cannot measure the wake factor that defined spacing (see below for an example). Many detected exceedances were for wakes residing less than 60 seconds in the corridor, which is less than the spacing normally allowed for runway landing and turnoff, and many exceedances were for wake residence times less than 5 seconds longer than predicted.

Although the AVOSS internally calculates wake constraints at individual approach windows, the final output specifies spacing criteria at top-of-approach, considering all wake prediction windows, runway occupancy time, and differences in assumed airplane speed along the common path. This feature allows system level studies that determine overall benefit due to system tradeoffs. The present AVOSS system has not been interfaced to an ATC system, but contains a simple model of runway arrival rate given the airport traffic mix (fraction of small, large, heavy aircraft), rounding of spacing criteria to manageable increments, and a nominal ATC delivery precision. The AVOSS output is designed for eventual interface to either the current ATC system, or to the Center-TRACON Automation System (CTAS). Details of the arrival rate model are provided in reference [6].

The objective of the development effort and demonstration is to develop the maturity level of each system to the point that concept feasibility can be shown in an operational environment, with all variables present, and that the technology is ready for operational testbed development for specific applications. The system demonstrated emphasizes the scientific validity of the weather profile measurements and wake predictions, and not the final engineering required for prototype operational equipment. As such, certain features such as system self-test and safety monitoring are implemented to the degree required for demonstration of the system concept. In the process of building a concept demonstration system, a powerful tool for system testing and tradeoff studies has been developed. Examples of the use of the AVOSS system for system design refinements and tradeoff studies will be provided.

\section{AVOSS System Enhancements and Performance Update}

An initial performance assessment [6] of AVOSS was provided in early 2000. That assessment was based on a year of meteorological subsystem data with no validating wake lidar data and a 13-day data set in the fall of 1999 with validating lidar data. The results showed the potential runway arrival rate increasing by about $6 \%$ on average, with as little as $3 \%$ increase on certain days and as much as $11 \%$ increase on favorable days. Since the benefit of a wake spacing system varies with the 
traffic mix, and the Dallas-Fort Worth International Airport (DFW) receives a high percentage of large aircraft which create no wake constraints for following large or heavy aircraft, the AVOSS spacing criteria was also applied to a traffic mix representing Miami, with a high fraction of heavy aircraft. The potential arrival rate increase with that traffic mix was about $12 \%$, or nearly double the DFW improvement.

A negative outcome of the initial assessment of the fall, 1999 lidar system deployment was poor automated wake prediction validation results. Although hand-examination of individual wake cases showed good agreement between wake predictions and observations, the automated comparison process falsely detected many exceedance events, defined as a wake persisting longer than predicted within the safety corridor. Of 423 buffer time calculations logged, the difference between predicted and observed wake time in the corridor, only $174(41 \%)$ resulted in positive buffers while 249 (59\%) produced exceedance events (negative buffer values). As reported [6], these results were due primarily to the simple comparison logic used. Many of the exceedance cases were logged when both the predicted and observed wake residence times were less than the possible spacing between aircraft. Other exceedances were logged when the wind line residence time, which is based only on wake drift, was compared to the predicted wake residence time based on wake sink at the same window. The wind line (or ground wind vortex sensing system) consists of a row of anemometers on poles oriented perpendicular to the flight path. Lateral drift of wakes can be measured once the wakes have settled into the anemometer array. The large number of exceedance cases logged in this first study prevented turnaround of that data to determine if any given exceedance was due to weather estimation, wake prediction, or wake sensing errors.

\section{Wake Comparison Logic Redesign}

The wake vortex comparison and validation logic has been redesigned for the final demonstration system. The major changes implemented include: (1) creation of "class 1" comparisons and (2) creation of "soft" and "hard" buffers. A class 1 comparison indicates that both the wake prediction and observation were less than a specified minimum time, currently set at 50 seconds. This time was chosen as the lowest possible aircraft spacing required for considerations such as runway occupancy time. Prediction buffers are not calculated for class 1 events, given the rationale that the wake was not a factor to following aircraft and the difference between, for example a 30-second residence prediction and a 40second observation, is not significant to operational safety.

The separate creation of soft and hard buffers was needed since each wake sensor does not always quantify each wake factor (sink, drift, demise) that governs aircraft separation. The ground wind line is only used for wake drift validation, since wake height and strength estimates from that system are in a very early stage of experimentation. Likewise, a lidar might track a wake but provide low confidence strength values in a particular case. In these situations a wake observation in perfect agreement with predictions may generate an exceedance detection. An example is a wake that sinks below the safety corridor floor in 20 seconds but drifts outside the lateral limits in 70 seconds. If the sensor only quantifies the 70second drift, a 50-second exceedance will be logged when comparing to the 20 -second, sinkbased residence time.

The revised comparison logic determines which of the three wake factors was used when calculating the aircraft spacing criteria, then determines if the wake sensor quantified that parameter while tracking. If the sensor quantified the factor used for spacing, then the calculated prediction buffer is "hard". If the sensor did not quantify the factor, then the prediction buffer is "soft". Since the wind line is 
only used for drift validation and is at a location dominated by the wake sink factor for spacing, all wind line comparisons are considered soft. A negative prediction buffer is called an exceedance, which is either a hard exceedance or a soft exceedance depending on the type of buffer. Hard exceedances are considered genuine. Soft exceedances may or may not be genuine and require detailed examination of data files for diagnosis.

\section{Revised AVOSS Performance and Validation Results}

The wake vortex data from the fall, 1999 deployment were rerun with the modified AVOSS software, now designated Version 2.5, to evaluate the revised wake comparisons software and overall system prediction performance. Also, additional performance data has been collected during preparations for the final system demonstration in July 2000. This additional data includes a month of open-loop operation (no wake lidars deployed) and 3 days of wake lidar validation in June, 2000.

\section{System Throughput Results}

Results from recent operations are consistent with previous results. In both the 1999 deployment and in recent operations, the average predicted runway throughput (maximum possible arrival rate) increased on average $5 \%$ with daily values that ranged from a low of about $2 \%$ to a high of about $11 \%$. The average dropped from the $6 \%$ seen with the previous software release of AVOSS due to the use of more conservative cross wind confidence intervals and possibly due to an additional 36 hours ( 72 half-hour periods) of weather data that passed modified weather quality criteria (described below) in the current software release. These throughput increases are based on AVOSS spacing criteria at the top of the approach, inefficiencies introduced by the various aircraft speeds on the common path, the assumed traffic mix at DFW (25\% small, $60 \%$ large, $10 \% \mathrm{~B} 757$, and $5 \%$ heavy), rounding of spacing criteria to $1 / 2$ nautical mile increments, and a 10 -second ATC delivery precision value. The low gain on certain days is consistent with the evolution of spacing criteria to the present values, indicating that current spacing criteria are sometimes required. The large increase in throughput during certain days is consistent with the improved airport arrival rates frequently seen during visual operations. While a 5 to $11 \%$ throughput gain may seem small, it can have significant impact on airport delays. With four landing runways in use, the DFW airport typically lands about 110 aircraft per hour at peak demand. A 5\% gain implies 5 or 6 aircraft on the ground in a one-hour period that otherwise would be accepting delays in the air. Other work [7] suggests that increases in throughput of 5 to 10 percent can reduce delays by 40 to 50 percent as demand approaches capacity.

\section{Wake Validation Results}

Rerun of the 1999 deployment with Version 2.5 software produced significantly improved comparison results. More wake files were processed in rerun than were available real-time for several reasons. Timing issues within the 1999 version of the real-time system caused some wake files to be missed, and an unintentional system configuration change caused an entire day of pulsed lidar data to be ignored during the deployment. The additional 36 hours of valid weather system operation also allowed more wake comparisons. The number of wake validation cases dramatically increased from 423 to 952 . Of these cases, 656 (69\%) produced class 1 events, indicating that both the wake prediction and the wake observation agreed that the wake vortex was not a factor to following aircraft that are separated by more than 50 seconds. Positive prediction buffers were observed in 244 cases or $26 \%$ of the observations, indicating that the wake residence time was less than the predicted bound. Since the AVOSS separation criteria are based on the worst-case wake motion in a range of possible 
wake tracks, not the expected mean behavior, a large fraction of the buffers are expected to be positive. Negative buffers, or exceedance cases, were detected 52 times, or in $5.5 \%$ of the events. All of these exceedances were soft.

Adding three days of wake data from June, 2000 produced no significant change to the overall statistics. Table 2 summarizes the results. A total of 1235 wake validation cases have been scored, showing that in $2 / 3$ of the events the wakes were not a factor to following aircraft, in $27 \%$ of the cases the wakes were a factor for a shorter period than used in the spacing calculations, and in 5\% the detected wakes lasted longer than predicted. No hard exceedances were observed in 1999, and 4 were observed in 2000. Of the hard exceedance cases, the longest wake residence time was 59 seconds and the exceedance values ranged from 2 to 9 seconds. All exceedances are reported, including 14 events of duration less than 5 seconds and an additional 12 events between 5 and 10 seconds duration.

One purpose of deriving the exceedance statistics is to diagnose system operation and focus future development. Exceedance events can be caused by at least three major factors: (1) weather parameter estimation, (2) wake vortex prediction, or (3) wake sensor tracking. While the analysis of these exceedance cases is continuing, the initial examination suggests that the most prevalent situations are the weather estimation and sensor factors, with a few prediction issues. Of the 66 exceedance cases, 42 were drift rate differences detected at the wind line, located 983 meters (3220 feet) from threshold. These events tended to exist in similar wind conditions, with cross wind 30minute mean values below $2 \mathrm{~m} / \mathrm{s}$ and very low crosswind standard deviation values between 0.02 and $0.24 \mathrm{~m} / \mathrm{s}$. For all cases, the average of the crosswind means was $1.4 \mathrm{~m} / \mathrm{s}$ and the average of the standard deviations was $0.1 \mathrm{~m} / \mathrm{s}$. In 9 of the cases, the crosswind uncertainty was so large that AVOSS was ignoring wake drift for spacing reductions and the exceedance detections were not related to the aircraft spacing values being provided. Wake drift is ignored whenever the crosswind mean minus the standard deviation is less than $1 \mathrm{~m} / \mathrm{s}$. This analysis of exceedance is one example of the use of the AVOSS system as a testbed for system refinements. Operational experience is suggesting that minor changes to the initial estimate of $1 \mathrm{~m} / \mathrm{s}$ for the wake drift lockout, or other wind confidence interval statistics, perhaps a function of atmospheric stability class, would provide for more robust wake drift predictions. The system provides the basis for determining the performance effects of modifying safety margins.

The remaining 26 exceedance cases were evenly distributed between the pulsed and continuous wave $(\mathrm{CW})$ lidar. The $\mathrm{CW}$ lidar scanned a location only 84 meters away from the threshold, where aircraft are typically about 20 meters ( 66 feet) above ground. Only wake lateral drift and decay are used at this location to affect spacing, and these exceedances were differences between predicted and observed wake decay. The average exceedance was 7

Table 2 - Wake Vortex Validation Results

\begin{tabular}{|l|c|c|c|c|c|}
\hline & $\begin{array}{c}1999 \\
\text { Number of } \\
\text { Cases }\end{array}$ & $\begin{array}{c}2000 \\
\text { Number of } \\
\text { Cases }\end{array}$ & $\begin{array}{c}\text { Total } \\
\text { Number }\end{array}$ & $\begin{array}{c}1999 \\
\text { Percentages }\end{array}$ & $\begin{array}{c}\text { Total } \\
\text { Percentages }\end{array}$ \\
\hline Wake cases & 952 & 283 & 1235 & & $67.8 \%$ \\
\hline $\begin{array}{l}\text { Class 1 } \\
\text { events }\end{array}$ & 656 & 181 & 837 & $68.9 \%$ & $26.9 \%$ \\
\hline $\begin{array}{l}\text { Positive } \\
\text { prediction } \\
\text { buffers }\end{array}$ & 244 & 88 & 332 & $25.6 \%$ & $5.3 \%$ \\
\hline Exceedances & 52 & 14 & 66 & $5.5 \%$ & \\
\hline
\end{tabular}


seconds, with a maximum of 18. Additional analysis will be required to determine if the error source is in the estimation of atmospheric turbulence, wake prediction, or sensor errors. Several tracks are highly suspect. In several cases the wake strengths were observed to decay to negligible values in less than a minute, then increase to significant strengths. In one case demise was never declared by the real-time tracking algorithms even through the longest wake track was 57 seconds, the generating aircraft was an MD-80, and the ambient headwind was over $8 \mathrm{~m} / \mathrm{s}$ (16 knots). Real-time tracking algorithms (in both the $\mathrm{CW}$ and pulsed lidar) are in an early stage of development and can be effected by high ambient winds, challenging wake tracking geometry, or unexpected high return signals due to lidar beam striking the aircraft or fog. Assuming that at least some of the exceedance cases are genuine, the data suggests manual examination of lidar data to focus on the events most likely to be genuine, then careful analysis of the ambient weather conditions to determine if specific conditions may lead to slower decay than expected and better estimate error bounds on decay prediction for optimization of system safety margins.

The pulsed lidar scanned an area about 1.7 $\mathrm{km}$ (5500 feet) from the runway, at a point where the aircraft are typically 105 meters (340 feet) above ground. Nearly all of these 13 exceedance cases were related to wake sink rate. Several were obvious sensor errors, as when the wake sank normally but the system continued to track as the next airplane arrived, falsely indicating that the original wake had risen back to glide slope. Nearly $1 / 2$ of the events were typified by high wind values, of 8 to $11 \mathrm{~m} / \mathrm{s}$ (16 to 22 knots), which can reduce the observed sink rate by accelerating the wake decay, by providing turbulent eddies that may temporarily slow the wake downward motion, or by providing turbulence that challenges the wake tracking algorithms. These cases were associated with rapid decay of the wakes.

Other cases suggested that a combination of tracking accuracy and the comparison logic are continuing to provide exceedance detections when the wake observation agrees with the prediction. The wake track from an MD-80 aircraft created an exceedance due to the wake vertical residence time at 15:25Z on November 16, 1999. The prediction was for insignificant drift, decay in 47 seconds, and sink below the corridor floor in 18 seconds. The lidar observed little drift, lost both wake tracks within 40 seconds due to decay, and saw both wakes sinking at the same rate. While the starboard wake sank below the corridor floor in 15 seconds, the initial height of the port wake was 13 meters (43 feet) higher and had not yet reached the corridor floor when its track was lost at 20 seconds elapsed time due to decay. This case illustrates the difficulty of providing automated wake validation data with imperfect measurements. The corridor was safe for the next aircraft in 40 seconds, but the fact that the port wake decayed before the vertical residence time could be quantified led to the exceedance detection. Some of the exceedance events from the pulsed lidar were likely valid. The present wake prediction algorithm does not quantify the uncertainty in sink rate due to vertically sheared environmental wind or turbulent eddies. Further analysis is required to bound this uncertainty and provide appropriate safety logic within AVOSS.

While analysis and discussion of exceedance cases is required for system refinements and safety analysis, one should not lose sight of the overall performance capabilities of the system. The AVOSS is currently running with minimally maintained, off-the shelf weather sensors, little self-test capability, and with weather data fusion and wake tracking algorithms that are only in their second generation and which have had only 3 to 4 weeks of integrated system testing for incremental refinements. Weather data is being collected at distances of over 2 miles from the wake measurement sites, and wake predictions are being made up to 30 minutes prior to the observation. No advance information of actual aircraft approach weight or speed is available. Little opportunity has been available for refinements to the internal safety buffers and system gains. Yet with all these variables present, there have been only 4 cases of hard 
exceedances being detected out of 1235 measurements, and none of these exceedances involved wakes lasting more than 59 seconds within the safety corridor. Of the 62 soft exceedances noted, well over half were related to drift rate at the wind line location, which is not the factor governing spacing at that location in most weather conditions. The remaining exceedances point to a combination of improved meteorological algorithms, improved real-time wake tracking algorithms, improved validation logic, and improved quantification of wake decay and sink uncertainty. This level of performance at an early stage in the development of a wake spacing system is a strong indication of the feasibility of the concept. Focused testbed operation and iterative system refinements will be needed to provide the performance and safety levels required for operational use. The current system provides a basis for system tradeoff studies and refinements, either in field operation or using replay of field data. Examples of this capability will be provided.

\section{Utility of AVOSS for Tradeoff Studies.}

Several examples of the use of AVOSS for design tradeoffs have been presented. When the logic for automated comparisons between wake predictions and observations was modified, batch replay of deployment data provided a rapid assessment of the overall effect of the change on prediction buffer statistics. The large number of drift exceedance in very light crosswind conditions with very low cross wind standard deviation statistics suggests minor changes to the parameters used to prevent using wake drift in such light cross wind conditions. Replay for deployment data with this change will allow assessment of both the throughput gain and exceedance changes as the cross wind uncertainty process is modified. During the process of evaluating the crosswind statistics, a configuration error was discovered. The cross wind data was providing variance, while the software was assuming standard deviation. The software was modified to convert the variance values to standard deviation. With many wind variance values less than unity, converting this data to standard deviation prior to use had the effect of increasing the wind confidence intervals. The effect on throughput performance is small while 9 of the 34 wind line drift exceedance cases in 1999 are eliminated.

A final example of recent system refinements based on test operations include changes to the weather quality criteria. Quality flags are provided in each wind, turbulence, and a thermal profile. AVOSS tests these flags to determine if the quality is acceptable for spacing reductions. If the test fails, then the current FAA spacing criteria is provided. The initial quality criteria required a specified fraction of the data from each sensor (radar profiler, sodars, etc.) to be valid as well as quality criteria flags provided by the data fusion software to be good. System operation in May of 2000 showed frequent application of the default FAA criteria. Examination showed marginal performance by certain sensors. Although the data at the required altitudes was available from alternate sensors, and the data fusion software was providing high quality assessments, the AVOSS quality criteria was failing the wind profiles. Examination of the data by project meteorologists concluded that the AVOSS criteria was too strict and was rejecting valid profiles. The software was modified to rely on the assessment of the profile quality provided by the data fusion software, which greatly improved overall performance.

Since the AVOSS output is top-of-approach spacing and runway throughput, as well as wake prediction buffer statistics, effects of parameter changes on total system performance with actual weather data can be studied. The uncertainty of wake sink rate in some turbulence conditions led to a simple study to determine the throughput changes in the 1999 deployment if AVOSS is operated with wake sink rates that are a specified fraction of the modeled sink rate. The 13 days of the deployment were rerun with $100 \%, 50 \%$, and $0 \%$ of the modeled sink rate being used in the spacing calculations. The normal run condition is $100 \%$. Use of $0 \%$ effectively disables the use of wake sink and requires lateral drift or decay to 


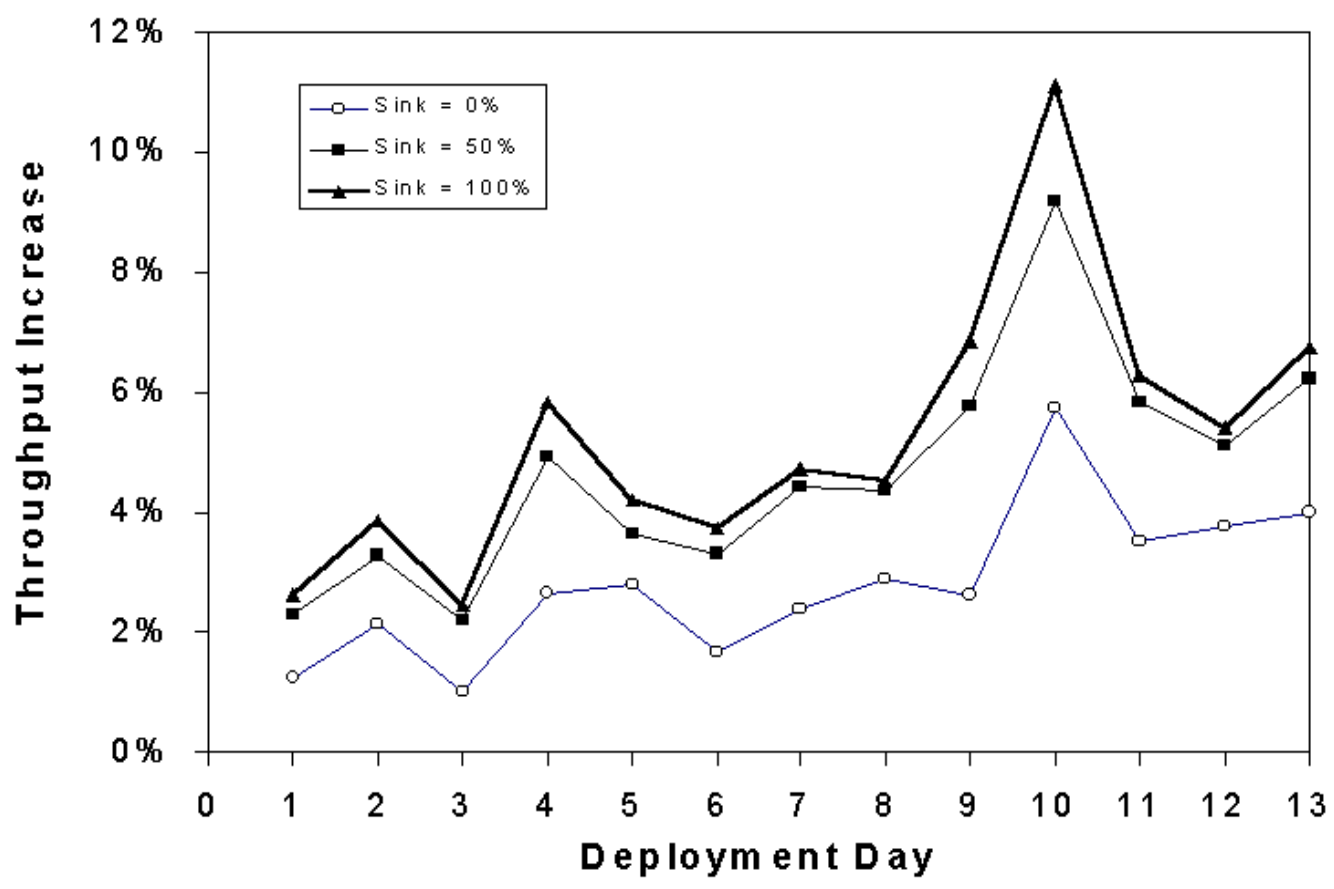

Figure 2 - Sensitivity Study of Throughput Increase to use of Wake Sink

reduce spacing. Figure 2 shows the results of these runs. Use of $50 \%$ sink rate had minimal effect on overall performance, while $0 \%$ sink rate substantially reduced performance, to about $1 / 2$ of that achieved with full use of wake sink modeling. This result suggests that wake sink rate uncertainty cannot be ignored, since elimination of the sink as a spacing reduction factor would seriously degrade performance, but that considerable uncertainty in the actual sink rate can be accommodated with minimal performance impact.

Other studies that are appropriate and feasible include assessment of methods and gains used to estimate uncertainty bounds for wake drift, sink, or demise, improved logic for automated wake validation, use of lidar wind data to reduce the weather subsystem part count, optimizing the aircraft data base to the traffic mix expected at the airport, dynamically altering prediction buffers by atmospheric stability class, and adaptation of the system to other applications.

\section{Application of AVOSS to Parallel Runway Operations}

An example of adaptation of AVOSS technologies to other applications is the closely spaced parallel approach at the San Francisco International Airport (SFO). The parallel runways $28 \mathrm{~L}$ and $28 \mathrm{R}$ are separated by only 229 meters (750 feet). During visual conditions, pilots fly simultaneous approaches, with one aircraft to the side and usually slightly behind the lead aircraft. In this position the wake vortex of the lead aircraft is not a factor, since it cannot cross the distance between the flight paths in time to effect the following aircraft. During conditions requiring instrument approaches, only one runway is available and airport delays are considerable. Concepts for allowing simultaneous parallel operations in certain instrument conditions are being assessed.

One such concept is the Simultaneous Offset Instrument Approach (SOIA) that offsets a localizer course to provide at least 762 meters 
(2500 feet) lateral separation until the offset aircraft can visually acquire the other and fly a conventional visual approach. The wake vortex issue is raised with this procedure, as it may be necessary to know the permissible interval behind each aircraft in which the trailing aircraft can be placed without risking a wake vortex encounter. The more rapid the wake drift between runways, the smaller will be the allowable interval between the landing aircraft. For example, with a wake drift rate of $5 \mathrm{~m} / \mathrm{s}$ (about 10 knots) the wake from the lead aircraft can cross to the other localizer in about 40 seconds, assuming it begins at the wing tip of a 29-meter (95-foot) semi-span aircraft. At an approach speed of $70 \mathrm{~m} / \mathrm{s}$ (about 140 knots) any aircraft trailing the lead by 2100 meters $(1.1 \mathrm{~nm})$ or more is at risk of an encounter.

The AVOSS system can be modified with minimal effort to provide this range information. The safety corridor currently used to predict the time required for a wake to exit the corridor of a single runway can be widened such that when the wake has exited that corridor it has become a threat to the parallel approach. The required software modifications to AVOSS are to modify code (that monitors wake drift) to consider the sign of the drift, that is whether the wake drifted to the right or the left, modify the code that parses window residence time into a spacing value to provide an allowable trailing distance value, and also provide a discrete indication when the wake of the lead is drifting away from the parallel approach and is therefore of no concern. Since the wind and wake vortex information is only needed at relatively low altitudes close to the airport, below about 366 meters (1200 feet) altitude, a single pulsed lidar situated to the side of the approach path would provide an excellent instrument for measuring the required wind and turbulence profiles, for wake predictions, and wake monitoring.

Such a self-contained AVOSS system was briefly tested at the John F. Kennedy International Airport (JFK) in 1998. This test took advantage of a NASA pulsed lidar test by installing a workstation inside the lidar trailer to run the AVOSS software. The lidar itself provided the crosswind and turbulence data required to run AVOSS, and then provided the wake vortex tracks for comparison. No weather inputs were provided by any sensor to the lidar system. It was only possible to make validating wake measurements at the lidar site, near the runway $31 \mathrm{R}$ middle marker. The lidar was located 627 meters along localizer and 375 meters right of localizer relative to the runway threshold. The lidar was tasked to provide actual wind measurements to an altitude of 115 meters, and then extrapolate the data above that altitude up to 600 meters for the purpose of allowing AVOSS to run without modifications. The intent of the test was to determine if a selfcontained AVOSS capability could be implemented at the lower approach altitudes using only the lidar as the sole source of weather data. System timing was the same as the full AVOSS system at Dallas, in that weather data is assimilated for 30 minutes, wake predictions are made from that 30-minute data set, and all wake observations are compared to that prediction set. This means that the wake comparison process is comparing observed wakes with those predicted using weather data from the previous 30-minute weather measurement period. This test approximates AVOSS architecture suitable for application to the SOIA concept or to departure spacing.

Although the AVOSS was initially operated from within the lidar trailer, subsequent AVOSS software improvements suggested that the deployment data be reprocessed with the now current AVOSS version 2.5. Initial results are very encouraging. During the 4-day deployment period, 203 wake tracks were collected. Of these, 94 occurred while valid wind profile and turbulence data were available. Valid aircraft identification was available for 47 of these wakes, which constitutes the wake data set that can be used for comparison with predictions. Of this 47-wake data set, 30 wake cases were either class 1 (both predicted and observed wake residence time less than 50 seconds), or produced positive prediction buffer values. Another 15 wake cases agreed with predictions, in that the prediction suggested that the wake would not leave the corridor, and the actual wakes were observed to remain within the 
corridor. AVOSS would provide current FAA spacing criteria in this instance. There were two cases of negative buffers, both hard (hard exceedances). One exceedance was for a duration of 5 seconds (84 seconds observed vs. 79 seconds predicted time) and the other was for a wake that lasted 50.3 seconds, or 0.3 seconds longer than allowed for cataloging as a class 1 event. Overall, this brief test suggests that a low-altitude AVOSS system can be operated from a self-contained lidar being used both for weather data collection and wake validation measurements.

\section{Summary}

The Terminal Area Productivity project has developed the technologies required (weather measurement, wake prediction, and wake measurement) to determine the aircraft spacing needed to prevent wake vortex encounters in various weather conditions. The system performs weather measurements, predicts bounds on wake vortex behavior in those conditions, derives safe wake spacing criteria, and validates the wake predictions with wake vortex measurements. System performance to date indicates that the potential runway arrival rate increase with AVOSS, considering common path effects and ATC delivery variance, is $5 \%$ to $12 \%$ depending on the ratio of large and heavy aircraft. The concept demonstration system, using early generation algorithms and minimal optimization, is performing the wake predictions with adequate robustness such that only 4 "hard exceedances" have been observed in 1235 wake validation cases. This performance demonstrates the feasibility of predicting wake behavior bounds with multiple uncertainties present, including the unknown aircraft weight and speed, weather persistence between the wake prediction and the observations, and the location of the weather sensors several kilometers from the approach location. A concept for the use of the AVOSS system for parallel runway operations has been suggested, and an initial study at the JFK International Airport suggests that a simplified AVOSS system can be successfully operated using only a single lidar as both the weather sensor and the wake validation instrument. Such a selfcontained AVOSS would be suitable for wake separation close to the airport, as is required for parallel approach concepts such as SOIA.

\section{References}

\section{Dallas-Fort Worth International Airport Short-Term Capacity Enhancement Plan, September, 1994.}

2. Hinton, David A.: Aircraft Vortex Spacing System (AVOSS) Conceptual Design, NASA TM-110184, August 1995.

3. Hinton, D.A.: An Aircraft Vortex Spacing System (AVOSS) For Dynamical Wake Vortex Spacing Criteria, AGARD 78th Fluid Dynamics Panel Meeting and Symposium on the Characterisation \& Modification of Wakes from Lifting Vehicles in Fluids, Trondheim, Norway, 20-23 May, 1996, AGARD CP-584, Paper 23.

4. Perry, R. Brad; Hinton, David A.; and Stuever, Robert A.: NASA Wake Vortex Research for Aircraft Spacing, AIAA 97-0057, 35th Aerospace Sciences Meeting \& Exhibit, January 9-10, 1997, Reno, NV.

5. Hinton, David A.; Charnock, James K.; Bagwell, Donald R.; and Grigsby, Donner: NASA Aircraft Vortex Spacing System Development Status, AIAA 99-0753, 37th Aerospace Sciences Meeting \& Exhibit, January 11-14, 1999, Reno, NV.

6. Hinton, David A.; Charnock, James K.; and Bagwell, Donald R.: Design of an Aircraft VOrtex Spacing System for Airport Capacity Improvement, AIAA-2000-0622, 38th AIAA Aerospace Sciences Meeting \& Exhibit, January 2000, Reno, NV.

7. Credeur, Leonard: Basic Analysis of Terminal Operation Benefits Resulting from Reduced Vortex Separation Minima, NASA TM-78624, October, 1977. 